\title{
Comparative study on the efficacy and safety of low-dose sodium valproate vs. diazepam in the prevention and treatment of pediatric febrile convulsion
}

\section{Wei He}

Department of Pediatrics, First People's Hospital of Fuyang District, Hangzhou, China

Correspondence to: Wei He. Department of Pediatrics, First People's Hospital of Fuyang District of Hangzhou City, Doctor's Office on the West 6th Floor of the Inpatient Department of the First People's Hospital of Fuyang District, No. 429 Beihuan Road, Fuyang District, Hangzhou 311400 , China. Email: wenfei1981@163.com.

Background: Febrile convulsion has a serious impact on the health and development of children. Diazepam treatment for febrile convulsion has many adverse reactions, which have a negative impact on the therapeutic effect. Therefore, the present study examined the efficacy and safety of low-dose sodium valproate on the treatment of pediatric patients with febrile convulsion.

Methods: From August 2016 to August 2019, a total of 110 children who suffered recurrent febrile convulsions were selected for the present study. These children were randomly divided into the observation group and the control group, with 55 children in each group. The control group was given low-dose diazepam therapy, while the observation group received low-dose sodium valproate. The therapeutic effects were compared between the two groups.

Results: The observation group had a significantly lower effective rate $(94.55 \%)$ than the control group $(74.55 \%, \mathrm{P}<0.05)$ and significantly shorter antispasmodic time and fever clearance time $(11.60 \pm 2.08 \mathrm{~min}$ and $11.39 \pm 1.81 \mathrm{~h}$, respectively) than the control group $(16.07 \pm 2.89 \mathrm{~min}$ and $17.09 \pm 3.12 \mathrm{~h})(\mathrm{P}<0.05)$. Six months after treatment, the observation group had higher scores on cognitive development indices than the control group, including gross motor skills, language, adaptability, fine motor skills, and social interaction $(\mathrm{P}<0.05)$. In addition, the observation group had a significantly lower incidence of adverse reactions and recurrence rate $(5.45 \%$ and $3.64 \%$, respectively) than the control group $(20.00 \%$ and $18.18 \%)(\mathrm{P}<0.05)$.

Conclusions: In the prevention and treatment of pediatric febrile convulsions, low-dose sodium valproate can rapidly relieve the clinical symptoms of children. In addition, this medication exhibits a high safety profile and significantly improves the cognitive ability of the patients. Low-dose sodium valproate has a definite therapeutic effect. Therefore, treatment with low-dose sodium valproate is worth promoting.

Keywords: Pediatric febrile convulsions; sodium valproate; diazepam; cognitive ability; safety

Submitted Jul 03, 2020. Accepted for publication Nov 24, 2020.

doi: $10.21037 / \mathrm{tp}-20-260$

View this article at: http://dx.doi.org/10.21037/tp-20-260

\section{Introduction}

Febrile convulsions occur in multiple neurological diseases. The incidence of febrile convulsion is highest in children aged 6 months to 3 years. As the age increases, febrile convulsions happen less often (1). The main symptoms of febrile convulsion in children include muscle spasm and fever. Moreover, the onset of this disease is acute, and the condition of this disease changes rapidly. If children with febrile convulsion are not treated quickly, the brain function may suffer irreversible damage as the disease progresses. The possibility of sequelae will increase, and the life and safety of the child may be threatened (2).

In the past, diazepam was frequently used to treat febrile convulsions in children, and it provided good control of 
the symptoms. However, there is a strict requirement on the blood concentration of diazepam. Inappropriate dosages of diazepam may lead to adverse reactions and affect the recovery of the children. As a broad-spectrum anticonvulsant, sodium valproate has high clinical value in the treatment of febrile convulsions in children. Lowdose sodium valproate has a significant control effect on adverse reactions when applied to treat pediatric patients with febrile convulsions (3). In addition, it rapidly alleviates the symptoms in the children, reduces the recurrence of the disease, and exhibits relatively high therapeutic efficacy and safety. The present study included 110 children who had recurrent febrile convulsions between August 2016 and August 2019 and examined the preventive and therapeutic effects of low-dose sodium valproate. The results are reported below.

We present the following article in accordance with the CONSORT reporting checklist (available at http://dx.doi. org/10.21037/tp-20-260).

\section{Methods}

\section{General information}

A total of 110 children who suffered recurrent febrile convulsions between August 2016 and August 2019 were selected and randomly divided into the observation group and the control group (55 patients per group). The control group consisted of 32 males and 23 females. The youngest child in the control group was 3 years old, while the oldest was 5 years (average age: $4.01 \pm 0.62$ years). In the control group, the lowest and the highest body temperature at the time of hospital admission were 37.6 and $41.2^{\circ} \mathrm{C}$, respectively (mean: $38.6 \pm 0.4{ }^{\circ} \mathrm{C}$ ), and the shortest and the longest course of disease were 2 months and 9 months, respectively (mean: $3.7 \pm 1.4$ months). The observation group consisted of 31 males and 24 females. The youngest child in the observation group was 3 years old, while the oldest was 5 years (average age: $3.98 \pm 0.65$ years). In the observation group, the lowest and the highest body temperature at the time of hospital admission were 37.8 and $41.1{ }^{\circ} \mathrm{C}$, respectively (mean: $38.8 \pm 0.3{ }^{\circ} \mathrm{C}$ ), and the shortest and the longest course of disease were 3 and 10 months, respectively (mean: $3.9 \pm 1.3$ months). All the children were diagnosed with febrile convulsions. This study was approved by the Ethics Committee of the First People's Hospital of Fuyang District, Hangzhou City (2020 Lunshen No. 007). The parents of each pediatric patient were informed about this study and agreed to participate. The study was conducted in accordance with the Declaration of Helsinki (as revised in 2013). Children with severe dysfunction of vital organs, neurological dysfunction, mental illness, allergies to the drugs used in the study, congenital diseases, traumatic brain injury, or contraindications to treatment were excluded. There was no significant difference in the general data between the two groups $(\mathrm{P}>0.05)$.

\section{Study methods}

After admission, all children were given conventional treatments such as anti-infection therapy, oxygen inhalation, maintenance of unobstructed breathing, correction of electrolyte disorders, nutritional support, and prevention of cerebral edema. In addition to the conventional treatments, the control group was given intravenous injection of diazepam at an initial dose of 40-200 $\mu \mathrm{g} / \mathrm{kg}$ (Tianjin King York Pharmaceutical Co., Ltd.; approval number: Guoyao Zhunzi H12020957). It was injected 3-4 times/d, and the daily dose did not exceed $10 \mathrm{mg}$. The dose was adjusted according to the changes in the children's symptoms and their response to the medication. The observation group was given the compound sodium valproate and valproic acid extended-release tablets at an initial dose of $10-15 \mathrm{mg} / \mathrm{kg}$ (Sanofi (Hangzhou) Pharmaceutical Co., Ltd.; approval number: Guoyao Zhunzi H20010595) on the basis of the conventional treatments. The dosage could be increased to $20-30 \mathrm{mg} / \mathrm{kg}$ according to the changes in the children's symptoms. Both groups were treated for 7 consecutive days.

\section{Observation indices}

The treatment effects were compared between the two groups. Full recovery was defined as the following: The clinical symptoms such as convulsions and fever disappeared completely after treatment, and no adverse drug reactions occurred. Marked effectiveness was defined as the following: the children's clinical symptoms were significantly improved after treatment, and no adverse reactions occurred. Effectiveness was defined as the following: The children's clinical symptoms were alleviated, and only mild adverse reactions developed. Ineffectiveness was defined as the following: The clinical symptoms of the children did not change or got worse after treatment, and many adverse reactions occurred. The total effective rate $=1-$ ineffective rate. 
Table 1 Comparison of the therapeutic effects between the two groups [ $\mathrm{n}(\%)]$

\begin{tabular}{|c|c|c|c|c|c|}
\hline Group & Fully recovered & Markedly effective & Effective & Ineffective & Total effective rate \\
\hline Control group $(n=55)$ & $23(41.82 \%)$ & $10(18.18 \%)$ & $8(14.55 \%)$ & $14(25.45 \%)$ & $41(74.55 \%)$ \\
\hline$\chi^{2}$ & - & - & - & - & 9.622 \\
\hline$P$ & - & - & - & - & $<\mathrm{fs} 0.05$ \\
\hline
\end{tabular}
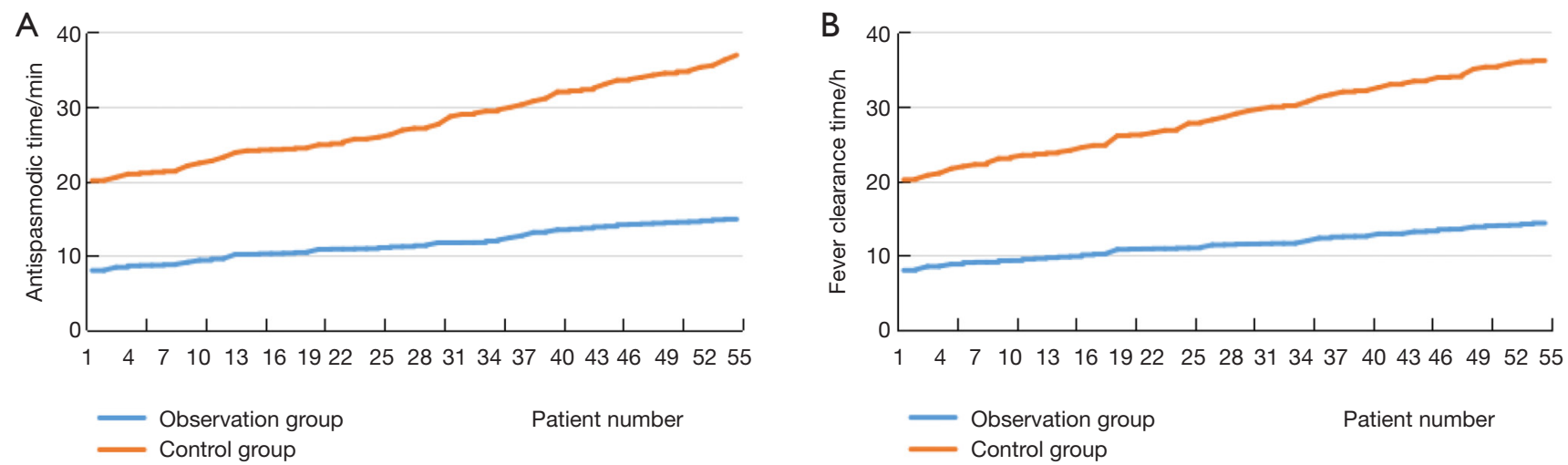

Figure 1 The comparative graphs of symptom relief time between the two groups of patients. (A) Antispasmodic time; (B) fever clearance time.

The antispasmodic time and fever clearance time were compared between the two groups. Before treatment and 6 months after treatment, the cognitive development of the two groups was scored using the Gesell Developmental Diagnostic Scale in terms of gross motor, language, adaptability, fine motor and social interaction, and the score range of each index was $0-130$ points. The higher the cognitive development ability, the better. The occurrence of adverse reactions such as blood pressure, gastrointestinal reactions, urinary retention, and respiratory depression during the treatment of the two groups and the recurrence of 6 months after treatment were compared.

\section{Statistical analysis}

Data were processed using the SPSS21.0 statistical software. A $P$ value $<0.05$ indicated that a difference was statistically significant. The measurement data were analyzed with Student's $t$-test and are expressed as the mean and standard deviation. The count data were analyzed by the $\chi^{2}$ test and are expressed as percentages.

\section{Results}

\section{Comparison of the curative effects between the two groups}

The total effective rate was $94.55 \%$ in the observation group and $74.55 \%$ in the control group $(\mathrm{P}<0.05$, Table 1$)$.

\section{Comparison of symptom relief time between the two groups}

The values of antispasmodic time and fever clearance time were sorted from small to large, and curves were plotted (Figure 1). Large differences can be seen in the antispasmodic time and fever clearance time between the two groups of patients. Further statistical analysis showed that both the antispasmodic time and fever clearance time were both shorter in the observation group than the control group $(\mathrm{P}<0.05$, Table 2$)$.

\section{Comparison of the cognitive development scores between the two groups before and after treatment}

Before the treatment, there were no significant differences 
in the scores of a number of cognitive development indices between the two groups, including gross motor skills, language, adaptability, fine motor skills, and social interaction $(\mathrm{P}<0.05)$. After treatment, all scores had increased in both groups $(\mathrm{P}<0.05)$, but the observation group had higher scores than the control group $(\mathrm{P}<0.05$, Table 3).

\section{Comparison of drug safety and recurrence between the two groups}

During the medication period, adverse reactions occurred in both groups of children. Among the children in the control group, four had hypotension, three had gastrointestinal reactions, two suffered urinary retention, and two suffered respiratory depression, for an incidence rate of $20.00 \%$ $(11 / 55)$. Among the children in the observation group, two had hypotension and one developed gastrointestinal reactions, for an incidence rate of $5.45 \%(3 / 55)$. The incidence rate of adverse reactions was significantly lower in the observation group $\left(\chi^{2}=7.213, \mathrm{P}<0.05\right)$. All the children had mild adverse reaction symptoms, and the symptoms disappeared after symptomatic treatment or withdrawal, and no other serious complications occurred. Six months after treatment, two children in the observation group suffered relapse, manifested as muscle cramps, resulting in

Table 2 Comparison of symptom relief time between the two groups

\begin{tabular}{lcc}
\hline Group & $\begin{array}{c}\text { Antispasmodic } \\
\text { time/min }\end{array}$ & $\begin{array}{c}\text { Fever clearance } \\
\text { time/h }\end{array}$ \\
\hline Observation group $(\mathrm{n}=55)$ & $11.60 \pm 2.08$ & $11.39 \pm 1.81$ \\
Control group $(\mathrm{n}=55)$ & $16.07 \pm 2.89$ & $17.09 \pm 3.12$ \\
$t$ & 3.248 & 3.094 \\
$\mathrm{P}$ & $<0.05$ & $<0.05$ \\
\hline
\end{tabular}

a recurrence rate of $3.64 \%(2 / 55)$. In contrast, 10 children in the control group suffered relapse, in which 4 cases of muscle cramps, 3 cases of fever, 3 cases of muscle cramps with fever symptoms, for a recurrence rate of $18.18 \%$ $(10 / 55)\left(\chi^{2}=7.309, \mathrm{P}<0.05\right)$. After all relapsed children were treated with sodium valproate sustained-release tablets, the symptoms gradually disappeared.

\section{Discussion}

Febrile convulsion in children is relatively common in clinical practice. It is a manifestation of abnormally excited nerve activity in the motor function area of the brain, mainly caused by acute infection of the peripheral nervous system or other factors, that results in local or systemic muscle contraction characterized by paroxysms. These are the symptoms of transient disorders of brain function (4). Children's nervous system is not yet fully developed, and their brain function is often poorly controlled or regulated. Even a very small stimulus may evoke a strong sense of excitement in the brain, resulting in a convulsion. Especially in children with fever, a body temperature of over $38{ }^{\circ} \mathrm{C}$ may lead to febrile convulsions. The children may show upturned eyes and body spasms (5). The shorter the symptoms last after the onset of the convulsion, the better the child recovers. If the symptoms cannot be alleviated in time, the prolonged disease duration will cause greater damage to the brain. These children are more likely to suffer sequelae. Moreover, the child's life may be threatened (6). Therefore, effective treatments should be given to the child after the onset of febrile convulsion to relieve their clinical symptoms.

At present, drugs are the main clinical treatment for the symptoms of pediatric febrile convulsion. The purpose is to reduce the damage to the child's brain, thereby achieving the therapeutic goals. The treatment is mainly carried out from the following steps: First, control the symptoms of convulsions; second, lower the body temperature of the

Table 3 Comparison of the cognitive development ability (scores) between the two groups before and after treatment

\begin{tabular}{|c|c|c|c|c|c|c|}
\hline Group & Time & Gross motor & Language & Adaptability & Fine motor & Social interaction \\
\hline $\begin{array}{l}\text { Observation } \\
\text { group }(n=55)\end{array}$ & After treatment & $93.56 \pm 3.84^{* \#}$ & $94.29 \pm 3.04^{\star \#}$ & $93.19 \pm 3.47^{\star \#}$ & $94.26 \pm 3.18^{\star \#}$ & $93.34 \pm 3.08^{\star \#}$ \\
\hline \multirow{2}{*}{$\begin{array}{l}\text { Control group } \\
(n=55)\end{array}$} & Before treatment & $82.85 \pm 5.51$ & $83.18 \pm 5.14$ & $83.04 \pm 5.22$ & $84.33 \pm 4.99$ & $84.62 \pm 4.77$ \\
\hline & After treatment & $89.27 \pm 4.49^{\star}$ & $90.12 \pm 4.30^{\star}$ & $89.07 \pm 4.13^{\star}$ & $90.25 \pm 3.87^{\star}$ & $90.11 \pm 4.04^{\star}$ \\
\hline
\end{tabular}

Compared with the scores before treatment. ${ }^{*} \mathrm{P}<0.05$; compared with the control group, ${ }^{\sharp} \mathrm{P}<0.05$. 
child; third, find and eliminate the cause of the infection; fourth, investigate the cause of the convulsion. Among these medications, diazepam is a commonly used anticonvulsant drug. Diazepam is a benzodiazepine anti-anxiety drug that has anticonvulsant, hypnotic, and sedative effects. Once the drug enters the human body, it inhibits the activity of neurons in the limbic system, reduces the stimulation of neuronal activity in the midbrain reticular structure, inhibits cortical arousal, and reduces abnormal nerve excitability in the brain's motor function area, thereby alleviating the symptoms of febrile convulsions in children $(7,8)$. However, clinical research has found that diazepam also inhibits the respiratory center. If the dosage is too high, this drug also causes the occurrence of complications such as hypotension and urinary retention. Therefore, the dosage of diazepam is strictly constrained in clinical practice (9). Sodium valproate is a broad-spectrum anti-epileptic drug. This drug does not contain nitrogen as a component and suppresses convulsions caused by a variety of factors. At present, in clinical studies, the mechanism of action of sodium valproate in anticonvulsant therapy has not yet been fully clarified, but most studies believe that sodium valproate can exert multiple therapeutic mechanisms to make patients presynaptic and post-synaptic $\gamma$-aminobutyric Increased acid transmission has an inhibitory effect on $\gamma$-aminobutyric acid transaminase, affects calcium ion and sodium ion channels, reduces the continuous attack of neurons, and inhibits the thalamic cortex loop; at the same time, in the synthesis of $\gamma$-aminobutyric acid, there is also a The participation of sodium valerate and its relationship with degrading enzymes can achieve the anticonvulsant effect, and can alleviate the myoclonic epilepsy, various types of minor seizures, mixed epilepsy, etc. that often occur in the human body (10). Oral sodium valproate is absorbed rapidly, and its metabolites are mostly distributed in the extracellular fluids. In the blood, most of the metabolites bind to plasma proteins. In clinical practice, sodium valproate is mainly used to treat epilepsy patients in whom other anti-epileptic drugs are ineffective. This drug exhibits an especially significant effect in the treatment of minor epilepsy. It is the first choice for treatment for absence seizures and primary grand mal seizures (11). In addition, sodium valproate has a certain effect against infantile spasms and benign infantile myoclonic epilepsy. A combination of sodium valproate and other antiepileptic drugs, such as ethosuximide, may achieve a better therapeutic effect on myoclonic epilepsy (12). Besides the anti-epileptic effects, sodium valproate also has significant therapeutic effects against febrile convulsions, mental retardation, dyskinesia, herpes-induced pain, porphyria, adrenal dysfunction, chorea, and alcohol withdrawal syndrome (13). As a broad-spectrum antiepileptic drug, sodium valproate is used in the treatment of children with febrile convulsion. It increases the concentration of $\gamma$-aminobutyric acid and inhibits abnormal nerve excitability in the brain's motor function area by reducing the degradation of $\gamma$-aminobutyric acid and increasing the synthesis of $\gamma$-aminobutyric acid, thereby relieving the local or systemic muscle spasms and achieving therapeutic effects against febrile convulsion $(14,15)$. In addition, sodium valproate causes fewer adverse reactions, and the main adverse reactions are gastrointestinal disorders, neurological disorders, and skin reactions. Administration of low-dose sodium valproate not only controls and relieves the clinical symptoms of the affected children but also effectively reduces the occurrence of adverse reactions and ensures the safety of this medication.

Here, the total effective rate of low-dose sodium valproate reached $94.55 \%$ in the treatment of pediatric patients with febrile convulsions. This rate was significantly higher than that of low-dose diazepam $(74.55 \%, \mathrm{P}<0.05)$. These results indicate that the low-dose sodium valproate better relieves the clinical symptoms (such as spasms and fever) and achieves a better therapeutic effect when used to treat febrile convulsions. The antispasmodic time and fever clearance time were lower in the observation group than the control group $(\mathrm{P}<0.05)$, indicating that treatment of febrile convulsions with low-dose sodium valproate can relieve the clinical symptoms rapidly and help the child recover. Six months after treatment, the scores of cognitive development indices, such as gross motor skills, language, adaptability, fine motor skills, and social interaction were all higher in the observation group than the control group $(\mathrm{P}<0.05)$, indicating that application of low-dose sodium valproate in treatment of pediatric febrile convulsions promotes the recovery and development of the children's cognitive ability. The underlying reason is that treatment with lowdose sodium valproate rapidly relieved the children's clinical symptoms and reduced the damage to their brain function. With the rapid relief of symptoms, the children's brain function recovered quickly, and their cognitive ability recovered accordingly. In terms of medication safety, the incidence of adverse reactions was $5.45 \%$ in the observation group vs. $20.00 \%$ in the control group $(\mathrm{P}<0.05)$, indicating that low-dose sodium valproate for pediatric patients with febrile convulsion causes fewer adverse reactions and is safer. The recurrence rate was significantly lower in the 
observation group (3.64\%) than the control group (18.18\%, $\mathrm{P}<0.05)$, indicating that low-dose sodium valproate for febrile convulsions effectively reduced the recurrence rate of convulsions and achieved a significant long-term therapeutic effect.

In summary, low-dose sodium valproate therapy for the prevention and treatment of pediatric febrile convulsion rapidly relieves the children's clinical symptoms and improves their cognitive development. In addition, the drug causes few adverse reactions and has a reliable curative effect. Therefore, treatment with low-dose sodium valproate is worth promoting.

\section{Acknowledgments}

Funding: None.

\section{Footnote}

Reporting Checklist: The author has completed the CONSORT reporting checklist. Available at http://dx.doi. org/10.21037/tp-20-260

Data Sharing Statement: Available at http://dx.doi. org/10.21037/tp-20-260

Conflicts of Interest: The author has completed the ICMJE uniform disclosure form (available at http://dx.doi. org/10.21037/tp-20-260). The author has no conflicts of interest to declare.

Ethical Statement: The author is accountable for all aspects of the work in ensuring that questions related to the accuracy or integrity of any part of the work are appropriately investigated and resolved. This study was approved by the Ethics Committee of the First People's Hospital of Fuyang District, Hangzhou City (2020 Lunshen No. 007). The parents of each pediatric patient were informed about this study and agreed to participate. The study was conducted in accordance with the Declaration of Helsinki (as revised in 2013).

Open Access Statement: This is an Open Access article distributed in accordance with the Creative Commons Attribution-NonCommercial-NoDerivs 4.0 International License (CC BY-NC-ND 4.0), which permits the noncommercial replication and distribution of the article with the strict proviso that no changes or edits are made and the original work is properly cited (including links to both the formal publication through the relevant DOI and the license). See: https://creativecommons.org/licenses/by-nc-nd/4.0/.

\section{References}

1. Sampathkumar P, Kannan KS. A comparative study of serum zinc levels in children with febrile seizures and children with fever without seizures in an urban referral hospital. Int J Contemp Pediatrics 2018;5:977.

2. Walz JC, Frey BN, Andreazza AC, et al. Effects of lithium and valproate on serum and hippocampal neurotrophin-3 levels in an animal model of mania. J Psychiatr Res 2008;42:416-21.

3. Kaboré A, Diallo A, Savadogo H, et al. Febrile Convulsions in Infants at the Pediatrics University Hospital Center Charles de Gaulle of Ouagadougou (Burkina Faso). Open J Pediatr 2018;8:199-206.

4. Tshimangani T, Pongo J, Bodi Mabiala J, et al. Pediatric Acute Severe Neurologic Illness and Injury in an Urban and a Rural Hospital in the Democratic Republic of the Congo. Am J Trop Med Hyg 2018;98:1534-40.

5. Odajima H, Mizumoto Y, Hamazaki Y, et al. Occurrence of convulsions after administration of theophylline in a large Japanese pediatric population with asthma. Pediatric Asthma Allergy Immunology 2003;16:163-73.

6. Friderichsen C, Melchior J. Febrile convulsions in children, their frequency and prognosis. Acta Pædiatrica 1954;43:307-17.

7. Kwak AR, Kim JS. Caregivers' knowledge, concerns and management of pediatric febrile convulsions. Child Health Nurs Res 2014;20:149.

8. Higuchi Y, Kubo T, Mitsuhashi T, et al. Clinical epidemiology and treatment of febrile and afebrile convulsions with mild gastroenteritis: A multicenter study. Pediatr Neurol 2017;67:78-84.

9. Gavrielov-Yusim N, Hoshen M, Singer SR, et al. The weight of MMRV-related febrile convulsions among other clinical factors contributing to febrile convulsions in children. Vaccine 2014;32:4954-9.

10. Mehta S, Tayabali S, Lachmann R. Valproate-induced hyperammonemia - uncovering an underlying inherited metabolic disorder: a case report. J Med Case Rep 2018;12:134.

11. Izadi Firouzabadi L, Geer K, Mead P, et al. Severe hyperammonaemic encephalopathy resulting from the overlap between hepatic and valproate encephalopathy. Postgrad Med J 2018;94:664. 
12. Moon JW, Kang JH, Kim HJ, et al. Risk factor of influenza virus infection to febrile convulsions and recurrent febrile convulsions in children. Korean J Pediatrics 2009;52:785-90.

13. Bromley RL, Baker GA, Clayton-Smith J, et al. Intellectual functioning in clinically confirmed fetal valproate syndrome. Neurotoxicol Teratol 2019;71:16-21.

14. Löscher W, Rating D, Siemes H. GABA in Cerebrospinal

Cite this article as: $\mathrm{He} \mathrm{W}$. Comparative study on the efficacy and safety of low-dose sodium valproate $v s$. diazepam in the prevention and treatment of pediatric febrile convulsion. Transl Pediatr 2020;9(6):768-774. doi: 10.21037/tp-20-260
Fluid of Children with Febrile Convulsions. Epilepsia 1981;22:697-702.

15. Wu WC, Huang CC, Chung HW, et al. Hippocampal alterations in children with temporal lobe epilepsy with or without a history of febrile convulsions: Evaluations with MR volumetry and proton MR spectroscopy. JNR Am J Neuroradiol 2005;26:1270-5. 